Relations industrielles

Industrial Relations

\title{
An Introduction to Management Science, by Teichroew Daniel, John Wiley \& Sons, New York, 1964, 713 pages.
}

\section{L.-René Parenteau}

Volume 23, numéro 3, 1968

URI : https://id.erudit.org/iderudit/027935ar

DOI : https://doi.org/10.7202/027935ar

Aller au sommaire du numéro

Éditeur(s)

Département des relations industrielles de l'Université Laval

ISSN

0034-379X (imprimé)

1703-8138 (numérique)

Découvrir la revue

Citer ce compte rendu

Parenteau, L.-R. (1968). Compte rendu de [An Introduction to Management Science, by Teichroew Daniel, John Wiley \& Sons, New York, 1964, 713 pages.] Relations industrielles / Industrial Relations, 23(3), 518-518.

https://doi.org/10.7202/027935ar

Tous droits réservés @ C Département des relations industrielles de l'Université Laval, 1968
Ce document est protégé par la loi sur le droit d'auteur. L’utilisation des services d'Érudit (y compris la reproduction) est assujettie à sa politique d'utilisation que vous pouvez consulter en ligne.

https://apropos.erudit.org/fr/usagers/politique-dutilisation/ 
Pour mieux prouver ces deux hypothèses, l'outeur décrit le développement historique et commente les implications quotidiennes de quelques problèmes ò la fois religieux et économiques contemporains à la lumière des valeurs et des normes propres ò un théologien. II cherche en plus ò les mettre en relation ovec la préoccupation d'éducation et d'action sociale que manifestent les différentes églises.

Cet ouvrage est le dernier d'une série de onze études débutées en 1949 par le a Federal Council of the Churches of Christ in America dont la préoccupation principale était de mettre en relation la morale chrétienne et les valeurs prônées dans notre vie économique. Les dix autres études complétant la série s'intitulent respectivement: Goals of Economic Life, The Organizationol Revolution, Social Responsibilities of the Businessman, American Income and Its Use, The American Economy - Attitudes and Opinions, Christian Values and Economic Life, Social Responsibility in Farm Leadership, Social Responsibilities of Organized Labor, Responsibility in Mass Communication and the Church os Employer, MoneyRaiser and Investor.

II semble donc, pour avoir une vue juste des positions soutenues dans cet ouvrage, qu'il soit préférable de lire les onze manuscrits composant l'étude.

\section{Jean SEXTON}

An Introduction to Management Science, by Teichroew Doniel, John Wiley \& Sons, New York, 1964, 713 pages.

L'enseignement des mathématiques a trop souvent négligé l'aspect pratique de ces sciences. L'étudiont apprend des formules, suit des raisonnements, étudie des théories mais n'en demeure pas moins perplexe devant un problème précis à résoudre. Les exemples et les problèmes que lui propose l'enseignement traditionnel sont trop théoriques ou trop globaux pour se prêter ò une véritable application de l'enseignement reçu.

L'ouvrage de Teichroew veut remédier à cette situation. On peut dire que l'ensemble des mathématiques est prêté expressément à la solution des problèmes couramment rencontrés dans le monde des affaires et de l'administration. La première partie de An Introduction to Management Science s'avère spécialement originale et démontre bien la préoccupotion de l'auteur. Cette partie approche la formulation des problèmes des affaires et donne les différentes techniques fournies par les mathématiques pour les résoudre efficacement. Les autres parties reprennent les grands chapîtres traditionnels des sciences mathématiques et les relient à des groupes particuliers de problèmes, tels la production, les finances, la mise en marché, l'organisation.

Nous croyons cependant que cet ouvrage serait d'autant plus efficace s'il était réservé ò des étudiants déjà fomiliers avec les mathématiques modernes, le calcul intégral et le calcul différentiel, ou encore s'il était réservé ò des personnes qui ont déjò quitté l'école et qui doivent maintenant passer de la théorie ò la pratique.

\section{L.-René PARENTEAU}

Managerial Attitudes and Performance, par Lyman W. Porter and Edward E. Lawler, Richard D. Irwin, Inc., Homewood, Illinois, 1968, 209 pages.

L'étude des rapports entre la satisfaction au travail et le rendement est demeurée un champ privilégié de la psycho-sociologie industrielle. Sur ce sujet on a entassé articles sur articles, volumes sur volumes, présentant des travaux dont les approches théoriques sont déficientes et les résultats souvent contradictoires. Le volume de Lawler et Porter est loin d'être du « réchauffé » et vient remédier à ces lacunes en replaçant le sujet dans un modèle dont les variables sont définies d'une manière opératoire. De plus, des liens précis sont établis entre elles permettant l'élaboration d'hypothèses faciles à vérifier à l'aide d'instruments connus et largement utilisés par les chercheurs et les praticiens en relations de travail. C'est là un «marioge de raison » entre l'effort théorique et la recherche empirique.

Les recherches portant sur les attitudes à l'endroit de la tâche à accomplir et le rendement des individus s'effectuent habituellement chez les travailleurs du rang et les employés de bureaux. Quelques chercheurs s'aventurent au premier échelon de la pyramide de l'autorité. Lawler et Porter ne se limitent pas seulement à ce niveau, mais étendent leur étude à celui des cadres intermédiaires de l'entreprise privée et des agences administrotives au sein de la fonction publique.

Entreprendre un tel travail à ce niveau sans reviser sérieusement l'appareil théorique 